\title{
Dados alarmantes sobre a notificação de sífilis congênita em uma capital do Norte brasileiro: um estudo transversal
}

\author{
Alarming data on the notification of congenital syphilis in a capital of the North \\ of Brazil: a cross-sectional study
}

Manoel Pereira Guimarães ${ }^{1}$ (D), Mateus de Sousa Rodrigues² (D), Leonardo Fernandes e Santana ${ }^{3}$ (D), Orlando Vieira Gomes ${ }^{4}$ (D), Karla Layse dos Santos Silva ${ }^{5}$ (D), Jauane Vilela Santos Gonçalves Matos $^{6}$ (D), Elaine Azevedo Soares Leal ${ }^{7}$ (D)

\begin{abstract}
RESUMO
Modelo do estudo: Estudo de prevalência. Objetivo do estudo: Descrever o perfil clínico e epidemiológico dos portadores de Sífilis Congênita entre Janeiro de 2010 a Junho de 2016 em uma capital do Norte brasileiro. Metodologia: Foi realizado um levantamento das fichas de notificação para infecção por sífilis congênita da Vigilância Epidemiológica em Rio BrancoAcre no período de Janeiro 2010 a Junho de 2016. Os dados foram analisados por meio do Statistical Package for Social Sciences (SPSS). Resultados: Foram notificados 189 casos de sífilis congênita precoce. Apenas 36,51\% $(n=69)$ receberam diagnóstico de sífilis gestacional antes do parto. Os casos evoluíram com 74,6\% de nascidos vivos, 15,3\% de natimortos, $6,3 \%$ de abortos e $2,1 \%$ de óbitos por sífilis congênita. A relação entre o Venereal Disease Research Laboratory (VDRL) materno e o desfecho dos casos sugere que filhos provenientes de mães com títulos $\geq 1: 16$ representam $82,2 \%$ das evoluções deletérias associadas $(p=0,045)$. Conclusão: A sífilis na gestação é uma patologia ainda subdiagnosticada durante a gravidez e, nesse estudo, foi mais frequente em filhos de mães no auge da menacme, de baixa escolaridade e moradoras da zona urbana. Apesar das medidas preventivas já implantadas, a sífilis congênita continua sendo um problema de saúde pública nesta capital do Norte brasileiro e deve continuar como alvo de estudos que gerem novas estratégias de prevenção.
\end{abstract}

Palavras-chave: Sífilis Congênita; Epidemiologia; Cuidado pré-natal; Doenças sexualmente transmissíveis.

\begin{abstract}
Study model: A Prevalence study. Objective: Describing the clinical and epidemiological profile of patients with Congenital Syphilis between January 2010 and June 2016 in a capital city in the North of Brazil. Methodology: A survey was carried based on the reports of infection for congenital syphilis of the Epidemiological Surveillance in Rio Branco-Acre from January 2010 to June 2016. The data were analyzed through the SPSS Program. Results: 189 cases of early congenital syphilis were reported. Only $36.51 \%(n=69)$ were diagnosed with gestational syphilis before delivery. The cases evolved with $74.6 \%$ of live births, $15.3 \%$ of stillborn infants, $6.3 \%$ of abortions, and $2.1 \%$ of congenital syphilis deaths. The relationship between the maternal VDRL and the outcome of the cases suggests that children from mothers with titles $\geq 1: 16$ represent $82.2 \%$ of the associated deleterious evolutions $(p=0.045)$. Conclusion: Congenital syphilis is a pathology still underdiagnosed during pregnancy and, in this study, was more frequent in children of mothers at the height of menacme, low schooling, and urban dwellers. Despite preventive measures already in place, congenital syphilis continues to be a public health problem in this capital of the Brazilian North and should continue to be the target of studies that generate new prevention strategies.
\end{abstract}

Keywords: Syphilis, Congenital; Epidemiology; Prenatal Care; Sexually Transmitted Diseases.

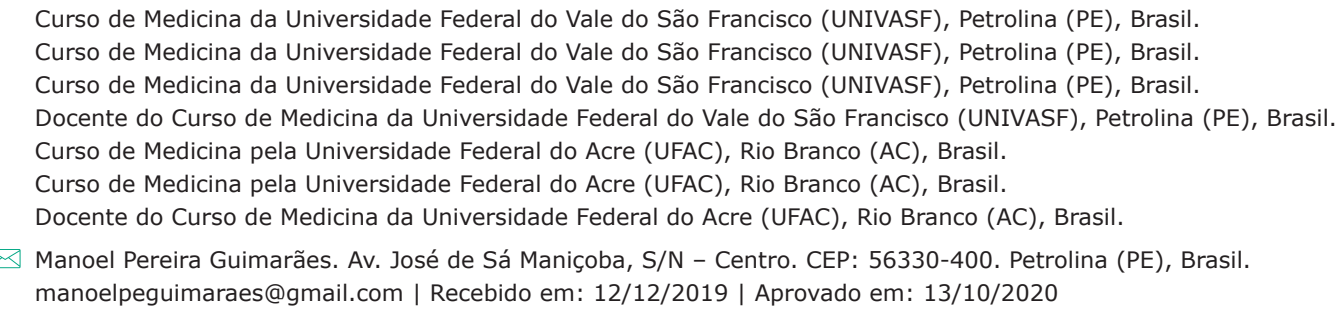




\section{INTRODUÇÃO}

A sífilis apresenta o Treponema pallidum como agente etiológico ${ }^{1}$ e acomete mais de dois milhões de gestantes por ano ${ }^{2,3}$. A sífilis na gestação é deletéria para o feto e possui um alto risco de transmissão vertical. Para fins de classificação, considera-se sífilis congênita precoce quando os pacientes apresentam até dois anos de idade, após essa faixa etária é considerado sífilis congênita tardia². O diagnóstico de sífilis congênita é realizado por uma avaliação epidemiológica criteriosa da situação materna e da avaliação clínico-laboratorial e exames de imagem na criança ${ }^{4}$. Cerca de dois terços dos nascidos vivos portadores de sífilis congênita são assintomáticos, no nascimento. Nos demais, as características clínicas variam conforme a classificação da doença. As taxas de transmissão vertical são de $70-100 \%$ nas fases primária e secundária. Já nas fases latente tardia e terciária da infecção materna, esse percentual reduz para cerca de $30 \%{ }^{5}$. Portanto, o diagnóstico precoce é fundamental.

Em 2007, o Brasil implantou o plano operacional para redução da transmissão vertical da sífilis. Esse plano possuía o Venereal Disease Research Laboratory (VDRL) como método de rastreamento de sífilis nas gestantes no primeiro e terceiro trimestres de gestação e, também, no momento do parto ${ }^{6}$. No ano de 2020 , foi atualizado o Protocolo Clínico e Diretrizes Terapêuticas para Atenção Integral às Pessoas com Infecções Sexualmente Transmissíveis (IST). De acordo com as diretrizes, persistiu a realização do teste rápido de sífilis em toda gestante que chega às unidades básicas de saúde como estratégia para diagnosticar a gestante com sífilis e tratar oportunamente ${ }^{7}$. Um bom acompanhamento pré-natal com realização de triagem durante a gestação e puerpério imediato e o tratamento adequado dos casos de sífilis gestacional constituem a base de prevenção e controle desta patologia. A sífilis na gestação é uma doença de notificação compulsória. A ocorrência de sífilis congênita é um marcador da qualidade de assistência à saúde materno-fetal, pois o risco de desfecho desfavorável para a criança diminui se a mãe tiver um diagnóstico e tratamento precoce e adequado ${ }^{8}$. Configura-se como tratamento adequado aquele que foi realizado 30 dias antes do parto com penicilina de dosagem variada, a depender do estágio da doença, e que também tratou o parceiro sexual da gestante ${ }^{7}$.

O Brasil apresentou um aumento significativo na incidência de sífilis congênita entre 2006 ( 2,0 casos/mil nascidos vivos) e 2015 ( 6,5 casos/ mil nascidos vivos) ${ }^{9}$. Entretanto, poucos são os estudos sobre sífilis congênita nas capitais do Norte do País. Isso dificulta a comparação dos dados desses locais com o cenário nacional.

Diante do exposto, o objetivo deste estudo é descrever o perfil clínico e epidemiológico dos portadores de Sífilis Congênita entre Janeiro de 2010 a Junho de 2016 em uma capital do Norte brasileiro.

\section{MATERIAL E MÉTODOS}

Trata-se de um estudo transversal, retrospectivo, descritivo, realizado mediante coleta de dados secundários a partir das fichas de notificação para infecção por sífilis congênita da Vigilância Epidemiológica em Rio Branco-AC, no período de Janeiro 2010 a Junho de 2016. A população de Rio Branco-AC é de aproximadamente 377.057 habitantes. Segundo o censo do Instituto Brasileiro de Geografia e Estatística (IBGE) de 2010, há aproximadamente 172.446 mulheres e 163.592 homens rio-branquenses, entre estes adultos e crianças ${ }^{10}$. A cobertura de saúde materno-infantil é realizada, basicamente, por duas maternidades, Hospital da Criança e 75 unidades municipais de saúde básica com atividades de pré-natal. A Maternidade e Clínica de Mulheres Bárbara Heliodora (MBH), assim como as unidades básicas, compõem a rede pública do município, sendo a MBH responsável, principalmente, pela demanda de alta complexidade no Estado; o Hospital Santa Juliana compõe uma rede mista (público-privada). A Maternidade Bárbara Heliodora é o principal polo de atendimento e conta com um fluxo de atendimentos por demanda espontânea e referenciada. Este serviço de atendimento é composto por ambulatório, urgência, Serviço Auxiliar Diagnóstico e Terapia-(SADT), regulação, internação e vigilância em saúde. Sua infraestrutura conta com uma média de 71 leitos $^{11 .}$

A amostra é composta por pacientes residentes de Rio Branco-AC, portadores de Sífilis Congênita Precoce - diagnosticados até o segundo ano de vida - 
notificados à Vigilância Epidemiológica em Rio Branco-AC. Foram excluídas as fichas de notificação de pacientes não provenientes de Rio Branco - AC e as notificações dos casos de sífilis congênita tardia (maiores de dois anos). Foi utilizado o banco de dados do Sistema de Informação de Agravos de Notificações (SINAN), para a busca dos casos de sífilis congênita. Após isso, os dados foram tabulados no Excel $®$ e submetidos à análise estatística por meio do Programa Statistical Package for Social Sciences (SPSS). As variáveis relacionadas ao perfil materno analisadas foram idade materna, escolaridade materna, zona de moradia, momento do diagnóstico, trimestre do diagnóstico e tratamento materno. Também foram estudados os sinais e sintomas apresentados pelos recém-nascidos com sífilis congênita.

O objetivo deste procedimento foi introduzir técnicas que permitam organizar, resumir e apresentar os dados para interpretação de acordo com os objetivos da pesquisa e o tipo de variável ${ }^{12}$. Os dados foram selecionados no sentido de compilar os quesitos essenciais para o cumprimento do objetivo do estudo. O banco de dados foi elaborado no Programa SPSS, em seguida foram realizadas análises de frequência, média e cruzamento de dados, buscando significância estatística através do teste Qui-quadrado. Os testes com $p<0,05$ foram considerados significativos. Para a discussão dos dados foram utilizados estudos realizados até o presente momento e materiais disponibilizados pelo Ministério da Saúde.
Esse estudo considerou como "tratamento adequado" o uso de penicilina benzatina, para tratamento de sífilis gestacional, em doses conforme indicação de cada fase clínica, assim como o concomitante tratamento do parceiro pelo protocolo do Ministério da Saúde.

Os dados foram obtidos mediante a autorização da Secretaria Municipal de Saúde e aprovação pelo Comitê de Ética e Pesquisa da Fundação Hospital Estadual do Acre (FUNDHACRE) sob o parecer no 1.547.570 (CAAE: 54187815.7.0000.5009). Este estudo segue a Declaração de Helsinque e está em conformidade com a resolução no 196/96 do Conselho Nacional de Saúde (CNS).

\section{RESULTADOS}

Foram notificados 189 casos de sífilis congênita precoce, em Rio Branco-AC, entre janeiro de 2010 e junho de 2016. O maior número de casos foi registrado em 2014 com um total de 57 casos, como mostra a Figura 1. O período de 2013 a 2015 respondeu por cerca de $77,24 \%$ dos casos.

Em relação ao perfil epidemiológico materno, $77,25 \%(n=146)$ das mães tinham entre 10 e 29 anos, $89,95 \%(n=170)$ eram provenientes da zona urbana da capital acreana e 43,39\% (n=82) apresentavam baixa escolaridade (até 7 anos estudados), como mostra a Tabela 1. Quanto ao grau de escolaridade, não foi possível avaliar 42 casos, pois a informação havia sido ignorada em tais fichas.

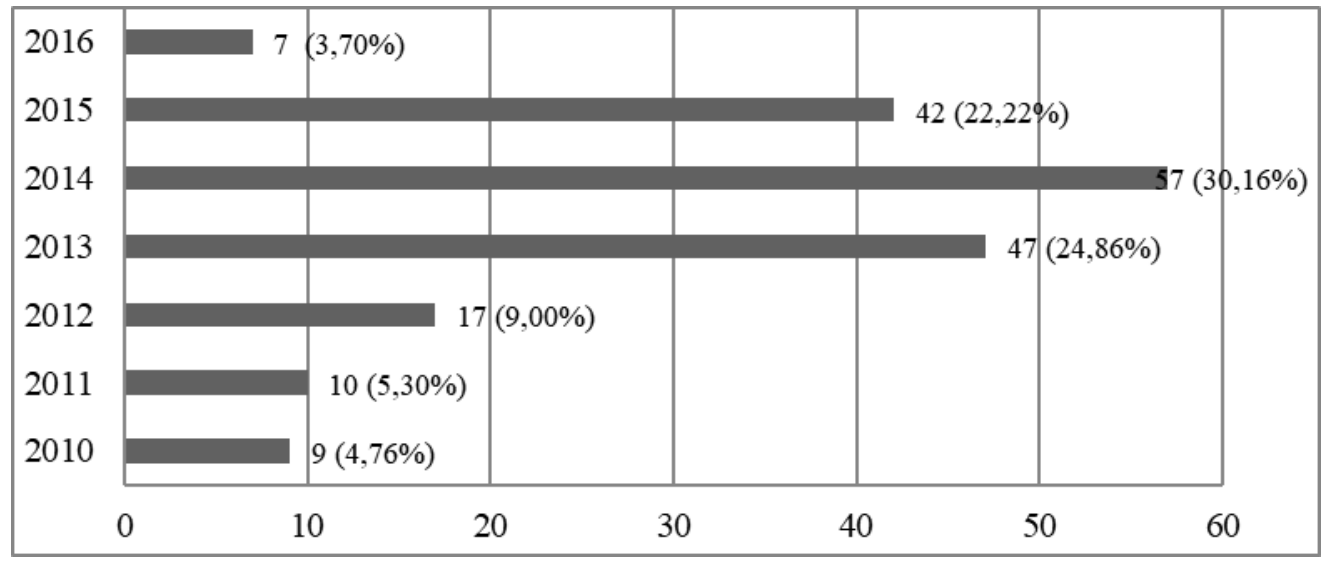

Figura 1. Número de casos de sífilis congênita por ano, em Rio Branco-AC, segundo ano de notificação (2010-2016).

Fonte: Sistema de Informação de Agravos de Notificação (SINAN) 
Tabela 1

Perfil Materno dos Casos Notificados para Sífilis Congênita, em Rio Branco (2010-2016).

\begin{tabular}{|c|c|c|}
\hline Variável & N Absoluto & $\mathrm{N} \%$ \\
\hline \multicolumn{3}{|l|}{ Idade Materna } \\
\hline 10 a 19 anos & 59 & 31,22 \\
\hline 20 a 29 anos & 87 & 46,03 \\
\hline 30 a 39 anos & 38 & 20,11 \\
\hline$\geq 40$ anos & 4 & 2,11 \\
\hline Não consta & 1 & 0,53 \\
\hline \multicolumn{3}{|l|}{ Escolaridade } \\
\hline Analfabeto & 4 & 2,12 \\
\hline Ensino fundamental incompleto & 69 & 36,51 \\
\hline Ensino fundamental completo & 9 & 4,76 \\
\hline Ensino médio incompleto & 28 & 14,81 \\
\hline Ensino médio completo & 33 & 17,46 \\
\hline Ensino Superior incompleto & 4 & 2,12 \\
\hline Ensino médio completo & 42 & 22,22 \\
\hline \multicolumn{3}{|l|}{ Zona de Moradia } \\
\hline Urbana & 170 & 89,95 \\
\hline Rural & 15 & 7,93 \\
\hline Periurbana & 4 & 2,12 \\
\hline \multicolumn{3}{|l|}{ Momento do Diagnóstico } \\
\hline Pré-natal & 69 & 36,51 \\
\hline Parto/Curetagem & 98 & 51,85 \\
\hline Após o parto & 16 & 8,46 \\
\hline Ignorado & 5 & 2,65 \\
\hline Não realizado & 1 & 0,53 \\
\hline \multicolumn{3}{|l|}{ Trimestre do diagnóstico } \\
\hline 10 trimestre & 11 & 5,82 \\
\hline 20 trimestre & 18 & 9,52 \\
\hline 30 trimestre & 63 & 33,33 \\
\hline Idade Ignorada & 3 & 1,59 \\
\hline Não consta & 94 & 49,74 \\
\hline \multicolumn{3}{|l|}{ Tratamento Materno } \\
\hline Adequado & 4 & 2,12 \\
\hline Inadequado & 165 & 87,3 \\
\hline Não realizado & 15 & 7,93 \\
\hline Não consta & 5 & 2,65 \\
\hline
\end{tabular}

Fonte: Sistema de Informação de Agravos de Notificação (SINAN)

No que diz respeito ao pré-natal, $76,2 \%$ $(n=144)$ das mães realizaram o acompanhamento (Tabela 1). Entretanto, apenas quatro $(2,12 \%)$ mães tiveram tratamento adequado. Esse estudo observou que dez mães não realizaram tratamento e 126 foram tratadas inadequadamente. Do total de casos analisados, apenas 36,51\% $(n=69)$ receberam diagnóstico de sífilis gestacional antes do parto, sendo $25,39 \%$ no terceiro trimestre. Quanto aos quesitos de adequação do tratamento, $86,8 \%$ dos parceiros não foram tratados.
Os casos evoluíram com $74,6 \%$ de nascidos vivos, $15,3 \%$ de natimortos, $6,3 \%$ de abortos e $2,1 \%$ de óbitos por sífilis congênita. A relação entre o VDRL materno e o desfecho dos casos sugere que filhos provenientes de mães com títulos $\geq 1: 16$ representam $82,2 \%$ das evoluções deletérias associadas $(p=0,045)$. As mães tratadas adequadamente não apresentaram nenhuma perda fetal ou óbito do concepto por sífilis congênita. No entanto, as mães que não realizaram o tratamento ou que o fizeram inadequadamente apresentaram $20 \%$ e $14,9 \%$ de perdas fetais ou óbito por sífilis congênita, respectivamente.

Quanto a sintomatologia, entre os nascidos vivos, $49,20 \%$ eram assintomáticos e $8,50 \%$ $(n=13)$ sintomáticos, os demais casos não tinham informações consistentes dessa variável. Isso significa que $42,30 \%$ das fichas continham falha de preenchimento nesse quesito. Dos casos sintomáticos, os sinais e sintomas relacionados às vísceras abdominais (icterícia, hepatomegalia e esplenomegalia) foram os mais frequentes, como mostra a Tabela 2.

\section{Tabela 2}

Sinais e sintomas de crianças com sífilis congênita, em Rio Branco (2010-2016).

\begin{tabular}{lc}
\hline \multicolumn{1}{c}{ Sinais e Sintomas } & $\begin{array}{c}\text { Número } \\
\text { de Casos }\end{array}$ \\
\hline Icterícia & 6 \\
Rinite Mucossanguinolenta & 2 \\
Anemia & 3 \\
Esplenomegalia & 5 \\
Hepatomegalia & 5 \\
Lesões cutâneas & 3 \\
Pseudoparalisia & 0 \\
Osteocondrite & 0 \\
Outro sintoma (plaquetopenia, púrpura) & 3 \\
\hline Fon: Sintema de
\end{tabular}

Fonte: Sistema de Informação de Agravos de Notificação (SINAN)

\section{DISCUSSÃO}

A notificação compulsória de sífilis congênita em todo o território nacional foi instituída e incluída no SINAN, desde $1986^{13}$. Foram notificados 408 casos de sífilis congênita entre os anos 2000-2016. Apesar da viabilidade das medidas preventivas, a sífilis congênita é um problema de saúde pública desafiador até os dias atuais. O levantamento desse estudo encontrou 189 casos em Rio Branco, 
durante os anos estudados. Isso representa mais da metade $(57,09 \%)$ dos casos no Estado do Acre e $2,74 \%$ de notificações da região Norte, cifras consideráveis ${ }^{9,14}$. A gravidade desses resultados é evidenciada quando se compara a realidade da capital acreana a um estudo recente que observou apenas 27 casos suspeitos de sífilis congênita em um Hospital Universitário Central de Coimbra, durante os anos 2003 a $2014^{15}$. Esse período é o dobro do período amostral do presente estudo. Apesar disso, revelou menos que 1/7 dos casos de Rio Branco. Essa discrepância evidencia a gravidade do problema da sífilis na capital estudada quando comparado a outras regiões.

Neste trabalho, o maior número de casos por ano foi observado em 2014, porém, a maior incidência nacional se dá em 2015. Nesse ano, o país apresentou um aumento absoluto de casos de aproximadamente $3873 \%$ em relação ao ano de 2010. Rio Branco é uma das capitais abaixo da média nacional $(5,8$ casos $/ \mathrm{mil}$ nascidos vivos). No entanto, ainda acima da meta preconizada pelo Comitê Regional para Validação da Eliminação da Transmissão Materno-Infantil de HIV e Sífilis, criado em 2014 pela Organização PanAmericana de Saúde (OPAS), que estabelece uma taxa de incidência de sífilis congênita de $\leq 0,5$ caso/ mil nascidos vivos. O crescente número de casos entre 2012 e 2015 está relacionado, provavelmente, a dois fatores principais: o aumento em 5,5 vezes o número de testes rápidos de sífilis distribuídos e o desabastecimento de penicilina benzatina no país em 2014, quando ocorreu o segundo pico de notificações desde o ano de $2005^{16}$. Levando-se em consideração que a penicilina benzatina continua a ser a única droga com efeito comprovado sobre o feto e que tratamentos com ceftriaxona não demonstraram efeitos superiores ao da penicilina, o aumento do número de casos pode estar diretamente ligado ao desabastecimento da droga ${ }^{17}$.

No que concerne ao perfil materno encontrado durante a análise das fichas, a idade média constatada nesse trabalho é semelhante ao estudo realizado por Saraceni et al. ${ }^{18} \mathrm{em}$ que mediana de idade foi 23 anos no Amazonas, Ceará e Rio Janeiro ${ }^{18}$. A maior parte dos registros é proveniente de zona urbana e atestam mães que não completaram - $1^{\circ}$ grau escolar. Esse perfil sociodemográfico é semelhante ao observado por Chaves et al. ${ }^{19}$ que analisaram sífilis congênita em um hospital do Rio
Grande do Sul ${ }^{19}$. A sífilis em gestantes tem sido relacionada ao baixo nível socioeconômico. Embora não seja uma doença restrita às camadas menos favorecidas, esses resultados sinalizam que pouca escolaridade e baixa renda podem ser marcadores importantes de pouco acesso aos serviços de saúde ${ }^{20}$. A análise de variáveis clínicas, associadas à saúde da mulher gestante, revela um percentual $(76,2 \%)$ de pré-natais abaixo da meta ${ }^{9}$. A meta prévia instituída pelo Ministério da Saúde é $95 \%$ de cobertura. Esse é outro fator de risco para a patologia estudada, segundo a literatura ${ }^{21}$. Ainda nesse eixo temático, os dados de diagnóstico permitem uma crítica não só ao número, mas também a qualidade do pré-natal realizado, já que a maior parte das mães teve diagnóstico no parto e das que tiveram diagnóstico no pré-natal a maioria consta como ocorrido no terceiro trimestre. Menos de 3\% das mães realizaram tratamento adequado. Aliado a isso, a quantidade de parceiros tratados é ínfima. Todos esses quesitos tiveram padrões semelhantes ao do estudo multicêntrico envolvendo Amazonas, Distrito Federal e Rio de Janeiro ${ }^{18}$.

Dados dos pré-natais como idade gestacional de início, número de consultas e disponibilidade de testes para rastreio de sífilis gestacional não puderam ser analisados neste estudo, pois não constam nas fichas. Apesar de serem parâmetros de grande valia na análise de qualidade dos acompanhamentos prestados nas unidades do município, a ausência desses dados não diminui a observação do descaso com que tem se tratado essa patologia, já que a mínima parcela de parceiros tratados evidencia o perigo de reexposição da gestante à contaminação e à possibilidade de uma gestação culminar em sífilis congênita. As falhas na assistência do pré-natal, a realização do diagnóstico tardio ou um tratamento inadequado, são fatores importantes e explicam os motivos pelos quais ainda existe um elevado número de casos de sífilis congênita ${ }^{13}$. Vale destacar também que as medidas de prevenção da doença são simples e de baixo custo, enquanto o tratamento de uma criança com sífilis congênita é bastante prolongado e oneroso ${ }^{5}$. A oferta de serviços de assistência pré-natal altera os desfechos das gestações e a sua ausência pode elevar a mortalidade perinatal em até cinco vezes ${ }^{20}$. Tal hipótese foi corroborada por esse estudo, mas não apresenta resultados de mesma grandeza, já que ao tentar 
correlacionar a realização de pré-natal ao desfecho dos casos, constatou-se 31 óbitos entre as 111 mães que realizaram pré-natal e 14 óbitos entre as que não realizaram, uma proporção aproximada de $1: 2$.

No que se refere à presença de sintomas, a grande parte dos eventos foram assintomáticos e os que apresentaram sinais e sintomas tiveram como mais prevalente a icterícia; resultado semelhante ao estudo realizado por Souza et al.22. No caso específico da neurossífilis, existe a indicação clínica de realização de teste VDRL no líquor de filhos de mães com sífilis não tratada ou tratada inadequadamente e nos casos de sintomatologia e VDRL reagente. Apesar da pequena quantidade de testes reagentes, observa-se a importância do rastreio de neurossífilis e eficiência da pesquisa com VDRL, pois, quando relacionados testes reagentes e não-reagentes com presença de alteração liquórica, somente um caso foi falso-negativo $(p=0,02)$. Apesar do diagnóstico de neurossífilis em três casos notificados, a droga utilizada para tratamento não foi a Penicilina G Cristalina; medicamento de escolha para o tratamento da neurossífilis pela capacidade de atravessar a barreira hemato-encefálica ${ }^{17}$.

Durante a coleta de dados, percebeu-se que a notificação através das fichas ainda apresenta algumas limitações. Alguns casos não puderam ser analisados por ter a informação ignorada. Não há informações suficientes para análise do seguimento dos recém-nascidos notificados, a ausência de informação quanto ao teste realizado após 18 meses demonstra que há falha no seguimento desses pacientes após o diagnóstico.

\section{CONCLUSÃO}

Apesar das medidas preventivas já implantadas, a sífilis congênita continua sendo um problema de saúde pública e deve continuar como alvo de estudos que gerem novas estratégias de prevenção. Pelos dados apresentados, pode-se inferir que, a nível local, a sífilis congênita também tem sido um agravo para o qual há diminuta dispensação de cuidados. Há maior risco para o agravo em filhos de mães no auge da menacme, de baixa escolaridade e moradoras da zona urbana. Há clareza quanto a dificuldade de alcançar metas de pré-natal, imprescindíveis ao bom prognóstico da gestação em relação ao agravo estudado, mesmo com a norma protocolar de rastreio e tratamento, há um abismo entre o real e o ideal. A sífilis congênita tem se constituído um espelho da qualidade do pré-natal e do binômio saúde materno-infantil em Rio Branco-AC. As principais limitações encontradas nesse estudo foram as falhas de notificação, certamente por ser uma base de dados secundários; houve grande número de perdas durante as análises que podem influenciar variáveis importantes do estudo em questão.

\section{REFERÊNCIAS}

1. Conceição HN da, Câmara JT, Pereira BM. Análise epidemiológica e espacial dos casos de sífilis gestacional e congênita. Saúde em Debate. 2019;43(123):1145-58.

2. Araújo MAL, Andrade RFV, Barros VL de, Bertoncini PMRP. Factors associated with unfavorable outcomes caused by Syphilis infection in pregnancy. Rev Bras Saúde Matern Infant [Internet]. 2019 Jun;19(2):411-9. Available from: http://www.scielo.br/scielo.php?script=sci_arttext\&pid=S1519-38292019000200411\&tlng=en

3. ORGANIZAÇÃO MUNDIAL DA SAUDE. Eliminação mundial da sífilis congénita: Fundamento lógico e estratégia para acção. Oms [Internet]. 2008;46. Available from: http://apps.who.int/iris/bitstream/10665/43782/4/9789248595851_por.pdf

4. Nunes PS, Zara AL de SA, Rocha DFN de C, Marinho TA, Mandacarú PMP, Turchi MD. Sífilis gestacional e congênita e sua relação com a cobertura da Estratégia Saúde da Família, Goiás, 2007-2014: um estudo ecológico. Epidemiol e Serv saude Rev do Sist Unico Saude do Bras. 2018;27(4):e2018127.

5. Costa CC da, Freitas LV, Sousa DM do N, Oliveira LL de, Chagas ACMA, Lopes MV de O, et al. Sífilis congênita no Ceará: análise epidemiológica de uma década. Rev da Esc Enferm da USP. 2013;47(1):152-9.

6. Araújo CL de, Shimizu HE, Sousa AIA de, Hamann EM. Incidência da sífilis congênita no Brasil e sua relação com a Estratégia Saúde da Família. Rev Saude Publica. 2012;46(3):479-86.

7. BRASIL Ministério da Saúde. Protocolo Clínico e Diretrizes Terapeuticas para Atenção às Pessoas com Infecções Sexualmente Transmissíveis (IST). Secr Vigilância em Saúde, Dep Doenças Condições Crônicas e Infecções Sex Transm. 2020;(0014125063):1-248.

8. Dos Reis GJ, Barcellos C, Pedroso M de M, Xavier DR. Diferenciais intraurbanos da sífilis congênita: análise preditiva por bairros do Município do Rio de Janeiro, Brasil. Cad Saude Publica. 2018;34(9). 
9. Ministério da Saúde BRASIL. Boletim Epidemiológico de Sífilis - 2016. Bol Epidemiológico [Internet]. 2016;47(35):29. Available from: http://portalarquivos. saude.gov.br/images/pdf/2016/outubro/31/2016_030_ Sifilis-publicao2.pdf

10. Instituto Brasileiro de Geografia e Estatística (IBGE). Brasil. [Acesso em: 02 Jun 2015]. Disponível em: https://cidades.ibge.gov.br.

11. Sistema de Informação de Agravos de Notificação (SINAN). Brasil. [Acesso em 29 Jun 2017]. Disponível em: http://portalsinan.saude.gov.br/sinan-net.

12. Mundstock E, Fachel JM, Camey AS, Agranonik M. Introdução à Análise Estatística Utilizando o SPSS 13.0. Cadernos de Matemática e Estatística Série B: Trabalho de Apoio Didático. Universidade Federal do Rio Grande do Sul, Porto Alegre Mai 2006.

13. Falavina LP, Lentsck $M H$, Mathias TA de F. Tendência e distribuição espacial de doenças infecciosas em gestantes no estado do Paraná-Brasil. Rev Lat Am Enfermagem. 2019;27.

14. Brasil. Ministério da Saúde. Departamento de Vigilância, Prevenção e Controle das IST, do HIV/ AIDS e das Hepatites virais. Indicadores e dados básicos da sífilis nos municípios brasileiros [Internet]. Secretária de vigilância em Saúde. Disponível em: http://indicadoressifilis.aids.gov.br.

15. Magalhães $M$, Basto $L$, Areia $A L$, Franco $S$, Malheiro $M E$, Afonso ME, et al. Sífilis na gravidez e sífilis congênita: realidade de um hospital universitário central português. TT - Syphilis in Pregnancy and Congenital Syphilis: Reality in a Portuguese Central University Hospital. Rev Bras
Ginecol Obs [Internet]. 2017;39(6):265-72. Available from: http://dx.doi.org/10.1055/s-0037-1603646

16. Motta IA, Rey I, Delfino DS, Morita MO, Gomes D, Pouzas T, et al. Sífilis congênita : por que sua prevalência continua tão alta? Rev Med Minas Gerais [Internet]. 2018;28(6):45-52. Available from: http://rmmg.org/exportar-pdf/2418/v28s6a09.pdf

17. Avelleira JCR, Bottino G. Sífilis: diagnóstico, tratamento e controle. An Bras Dermatol. 2006;81(2):111-26.

18. Saraceni V, Fernando Mendes Pereira G, Freitas da Silveira M, Alix Leite Araujo M, Espinosa Miranda A. Vigilância epidemiológica da transmissão vertical da sífilis: dados de seis unidades federativas no Brasil. Pan Am J Public Heal [Internet]. 2017;41(1):1-8. Available from: http://portalsinan.

19. Chaves J, Bassani DC, Ghignatti B, Derlan CB, Koepp J, Possuelo L. Sífilis congênita: análise de um hospital do interior do estado do RS. Rev AMRIGS. Porto Alegre. 2014; 58 (3):187-192.

20. Magalhães DM dos S, Kawaguchi IAL, Dias A, Calderon I de MP. Sífilis materna e congênita: ainda um desafio. Cad Saude Publica. 2013;29(6):1109-20.

21. Guinsburg R, Santos AMN dos. Critérios Diagnósticos e tratamento da sifilis congênita. Soc Bras Pediatr. 2010;1-17.

22. Souza LFM, Monteiro PM, Mota A dos S, Junior ENP, Passos MRL. Analysis of congenital syphilis cases notification in a reference hospital of niteroi, Rio de Janeiro state 2008 to 2015. J Bras Doenças Sex Transm. 2017;29(1):2177-8264. 